\title{
Tracking motion of topological defects in a stripe charge-ordered phase with continuously variable temperature cryo-STEM
}

Noah Schnitzer ${ }^{1}$, Berit Goodge ${ }^{2}$, Elisabeth Bianco ${ }^{3}$, Alemayehu Admasu ${ }^{4}$, Jaewook Kim ${ }^{4}$, Sang-Wook Cheong $^{5}$, Ismail El Baggari ${ }^{6}$ and Lena Kourkoutis ${ }^{2}$

${ }^{1}$ Department of Materials Science and Engineering, Cornell University, United States, ${ }^{2}$ School of Applied and Engineering Physics, Cornell University, United States, ${ }^{3}$ Kavli Institute at Cornell, Cornell University, Ithaca, New York, United States, ${ }^{4}$ Department of Physics and Astronomy, Rutgers University, United States, ${ }^{5}$ Rutgers Center for Emergent Materials, Rutgers University, United States, ${ }^{6}$ The Rowland Institute at Harvard University, United States

Understanding the complex phase diagrams of quantum materials requires careful characterization and in situ control at the atomic scale. Scanning transmission electron microscopy (STEM) provides a powerful local probe which can measure structural modulations, such as the periodic lattice distortions (PLDs) associated with charge ordering, with high resolution and precision. The strong temperature dependence of many strongly correlated phenomena makes cryogenic temperature control key to studying the emergence, interaction, and transitions of these phases [1,2]. Using a recently developed side-entry holder which enables stable STEM imaging at temperatures from $\sim 100-1000 \mathrm{~K}$ [3], we have measured the PLDs in a charge ordered manganite from the low temperature commensurate phase to just below $\mathrm{T}_{\mathrm{c}}$, near room temperature. The holder's high stability and fast temperature control enable tracking of the same region of interest through complete temperature cycles, allowing the picometer scale displacements of every atomic column in the field of view to be measured at each temperature step. This dynamic information reveals how topological defects in the PLDs travel through the material and how the PLDs restructure around the defects as the temperature is cycled between near-room temperature and cryogenic temperatures $(\sim 100 \mathrm{~K})$.

$\mathrm{Bi}_{35} \mathrm{Sr}_{.17} \mathrm{Ca}_{.48} \mathrm{MnO}_{3}$ (BSCMO) is a model charge ordered system with a $\mathrm{T}_{\mathrm{c}}$ just above room temperature. Near room temperature, the wavevector of the PLDs associated with charge ordering is incommensurate, while cooling to liquid nitrogen temperature the wavevector lengthens to the commensurate position. Previous STEM work on the system showed that even at room temperature the PLD is locally commensurate at nanometer length scales, and the incommensuration is driven by phase inhomogeneity which manifests through topological defects and phase shifts in the PLD [4,5]. Here, we measure the displacements of the atomic columns associated with the striped PLD at several discrete temperature steps between 100 and $298 \mathrm{~K}$ over the same region of interest, revealing motion of topological defects upon heating. Figure 1 shows a topological defect in the PLD in a region of the sample that is otherwise free of crystalline defects. As the temperature of the sample is increased the topological defects present in the PLD move through the lattice causing a local restructuring of the PLD stripes; however, away from the defects the PLD remains almost entirely static. This is shown in Figure 2, which visualizes how the PLD stripes shift over 3 temperature steps. These findings suggest that the motion of topological defects may be a mechanism through which phase inhomogeneity in the PLD is propagated.

This work was supported by NSF (DMR-1719875, DMR-1539918, DMR-1429155) and AFOSR (FA 955016-1-0305). 


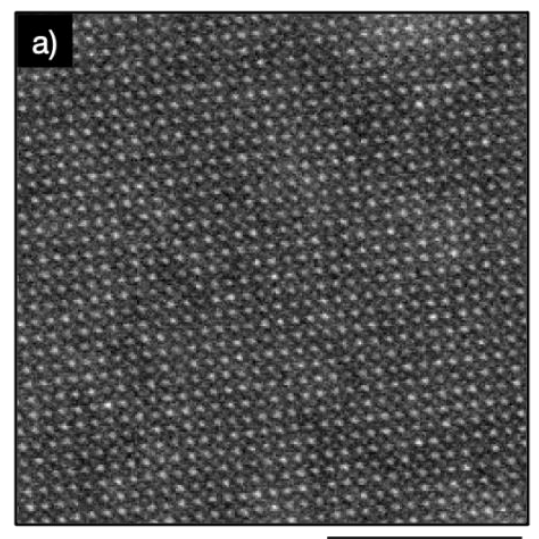

$5 \mathrm{~nm}$

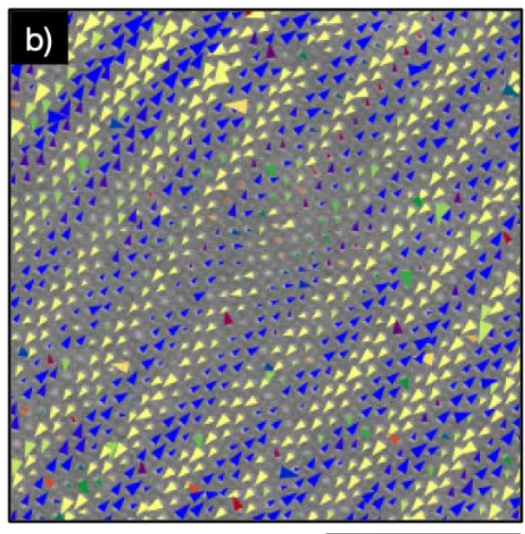

$\triangle 4 \triangle 6 \triangle 8 \quad \Delta 10$ $\Delta(\mathrm{pm})$

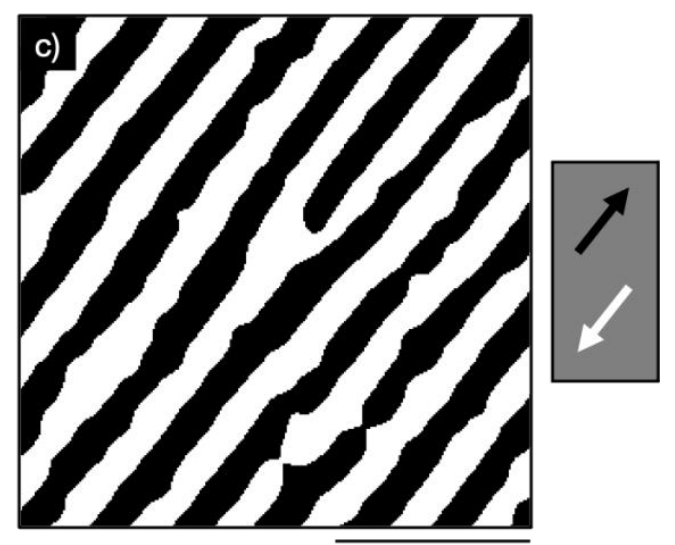

Figure 1. Measurement of a topological defect in a periodic lattice distortion (PLD) at $\sim 115 \mathrm{~K}$. a) Atomic resolution HAADF-STEM image shows a lattice free of crystalline defects. b) Map of picometer scale displacements of each atomic column in the low temperature phase reveal a topological defect in the PLD stripes. c) The defect and alternating directions of the stripes are highlighted by smoothing and thresholding the displacements from (b).
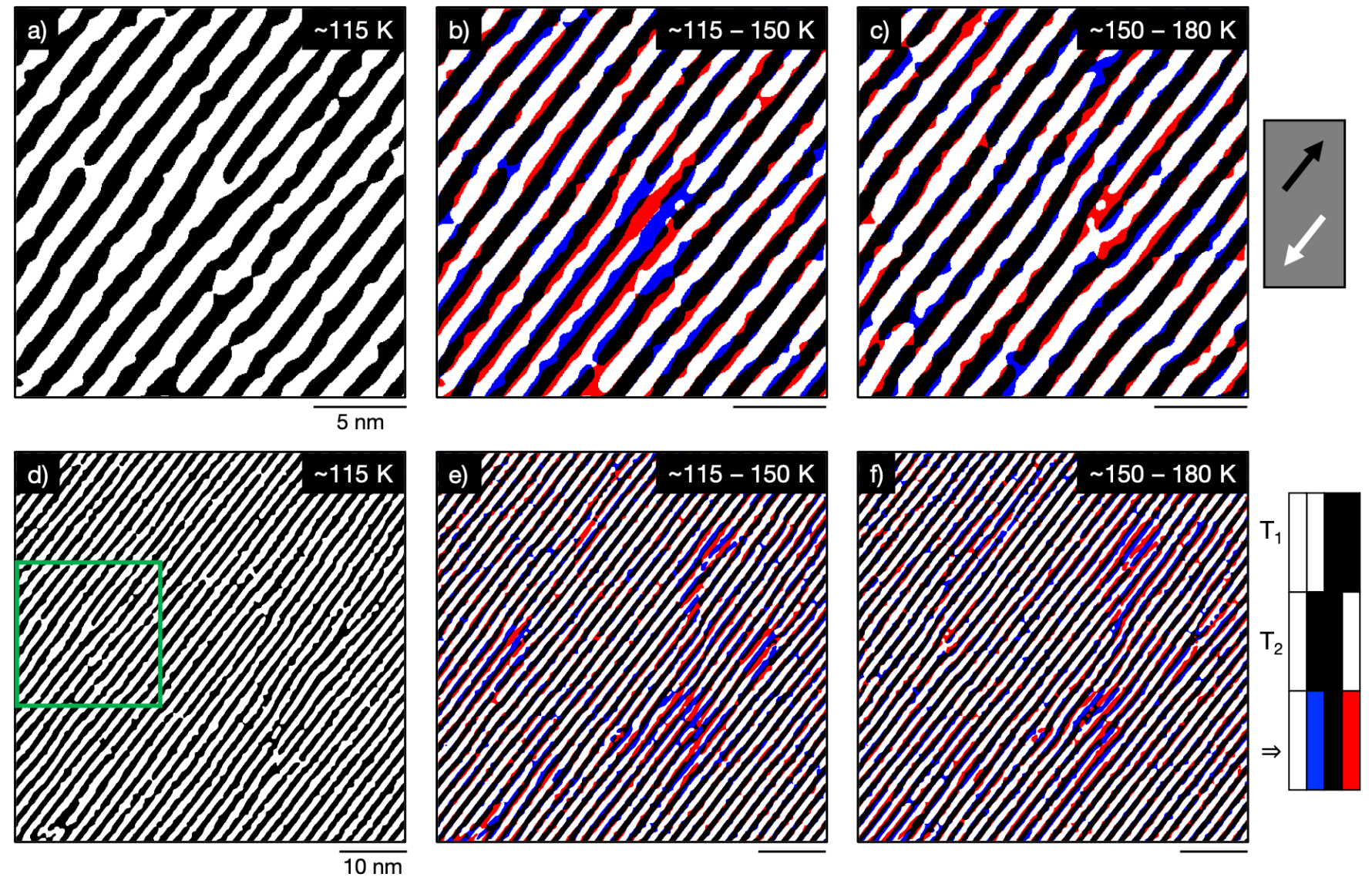

Figure 2. Motion of topological defects in PLD through temperature. a,d) Visualization of PLD stripes at $\sim 115 \mathrm{~K}$. Green box in (d) denotes the field of view shown in (a-c). b,e) Changes in stripes between 115 and $\sim 150 \mathrm{~K}$. e,f) Changes in stripes between $\sim 150$ and $\sim 180 \mathrm{~K}$. Regions shown in blue reversed direction 
from white to black, regions shown in red reversed direction from black to white upon heating, as shown in schematic on right. Away from the defects, the PLD remains largely unchanged across all temperatures.

References

[1] G. C. Milward, M. J. Calderón, P. B. Littlewood. Nature 433, 607-610 (2005).

[2] J. Chang, et al. Nature Physics 8, 871-876 (2012).

[3] B. H. Goodge, et al. Microscopy and Microanalysis 26, 439-446 (2020).

[4] B. H. Savitzky, et al. Nature Communications 8, 1833 (2017).

[5] I. El Baggari, et al. PNAS 115, 1445-1450 (2018). 